(2)

\section{OPEN ACCESS}

\title{
A Graceful EXIT impeded by obstetrical complications
}

\author{
Michael Zaretsky, ${ }_{1}^{1}$ Megan Brockel, ${ }^{2}$ Sarkis Christopher Derderian, ${ }^{3}$ Christian Francom, ${ }^{4}$ \\ Cristina Wood ${ }^{2}$
}

Department of Obstetrics

and Gynecology, University of Colorado Denver School of Medicine, Aurora, Colorado, USA ${ }^{2}$ Department of Anesthesia, University of Colorado Denver School of Medicine, Aurora, Colorado, USA

${ }^{3}$ Department of Surgery, University of Colorado Denver School of Medicine, Aurora, Colorado, USA

${ }^{4}$ Department of Otolaryngology, University of Colorado Denver School of Medicine, Aurora, Colorado, USA

\section{Correspondence to}

Dr Michael Zaretsky;

michael.zaretsky@

childrenscolorado.org

Accepted 17 December 2020

Check for updates

(c) BMJ Publishing Group Limited 2021. Re-use permitted under CC BY-NC. No commercial re-use. See rights and permissions. Published by BMJ.

To cite: Zaretsky M, Brockel M, Derderian SC, et al. BMJ Case Rep

2021:14:e237911.

doi:10.1136/bcr-2020-

237911

\section{SUMMARY}

We report an ex utero intrapartum therapy-to-airway procedure in which obstetric factors dramatically influenced the sequence of events necessary to complete the procedure.

\section{BACKGROUND}

Successful ex utero intrapartum therapy (EXIT) procedures are predicated on several factors, including a highly skilled and collaborative multidisciplinary team, optimal maternal and fetal anaesthesia management, maximum uterine quiescence and relaxation with maintenance of uterine volume, creation of a haemostatic hysterotomy with adequate exposure, real-time fetal monitoring, successful fetal intervention and, lastly, a seamless transition to neonatal intensive care and definitive surgical therapy. ${ }^{1-4}$ While fetal outcomes following an EXIT procedure have been well described, lesser emphasis has been placed on maternal obstetrical considerations that may heavily impact the successful completion of the procedure. We therefore report an EXIT-to-airway procedure in which obstetrical complicating factors dramatically altered the sequence of events necessary to complete the procedure.

\section{CASE PRESENTATION}

A 36-year-old gravida 5 para 3013 was referred to our fetal treatment centre at 24 weeks of gestation with a fetal diagnosis of congenital high airway obstruction syndrome (CHAOS) without additional anomalies (figures 1 and 2). Fetal imaging revealed a gap in the trachea, but sufficient tracheal length was present to permit access for tracheostomy. Fetal cytogenetics from an amniotic fluid sample revealed a normal karyotype and microarray. In a multidisciplinary conference involving the family, options were discussed to include termination of pregnancy, delivery with neonatal comfort care and an EXITto-airway procedure. The family was well informed that without an established airway at the time of delivery, a neonatal demise would be imminent. Fetoscopic laser decompression of CHAOS, feasible in select cases, ${ }^{5}$ was not considered in this scenario secondary to the long gap defect noted on fetal imaging, which would result in a low likelihood of a successful procedure, as well as the associated risks of surrounding tissue injury. Prematurity is also higher following fetoscopic procedures, and an EXIT procedure remains necessary regardless of decompression to develop an adequate airway at delivery. The family elected for a re-evaluation in the third trimester and returned at 31 weeks of gestational age for a repeat ultrasound and fetal MRI. She received weekly ultrasounds at the referring centre to determine fetal viability, monitor amniotic fluid volume and assess for signs of premature labour until she relocated to our centre. Findings on follow-up remained stable when compared with her earlier evaluation, with the exception of asymptomatic polyhydramnios.

After follow-up counselling, the family elected to undergo an EXIT-to-airway procedure scheduled for 37 weeks of gestation. At this point, a call tree was established placing all key specialties on 24-hour availability until the procedure was completed. The gestational age chosen for the procedure was established to allow for further fetal growth and to promote fetal lung maturity.

At 34 weeks 5 days of gestation, she presented to labour and delivery at 03:00 on a Sunday morning with the complaint of gross rupture of membranes. On examination, her cervix was noted to be $4 \mathrm{~cm}$ dilated; there was gross pooling of amniotic fluid; and the umbilical cord was prolapsed through the cervix up to the level of the introitus (figure 3). The fetus was in the breech presentation. Contractions were occurring regularly at $4 \mathrm{~min}$ intervals with persistent variable fetal heart rate decelerations down to 80 beats $/ \mathrm{min}$. The call tree was activated. The patient was taken to the operating room and underwent general anaesthesia with endotracheal intubation, while the multidisciplinary team congregated to discuss adjustments to the procedure. The time from patient presentation in labour and delivery triage to induction of general anaesthesia was 59 min, with all essential team members present.

The decision was made to make a maternal midline laparotomy to provide sufficient access to completely exteriorise the late-preterm gravid uterus and to make a fundal hysterotomy to deliver the fetal head and neck first. The placenta was anterior, and the fetus was in the breech presentation in the setting of anhydramnios secondary to rupture of membranes. An attempt at external cephalic version was not deemed safe in the setting of anhydramnios, active labour and existing cord prolapse. For these reasons, the original plan to perform a maternal abdominal transverse skin incision with a low transverse uterine hysterotomy was therefore abandoned. The midline skin incision was made to just below the umbilicus with placement of a large Olexis O retractor (Applied Medical, Rancho Santa Margarita, Californis, USA) allowing the uterus to be exteriorised. The anaesthesiologist provided maximal halogenated agents with a mean alveolar concentration of 3 , along with 


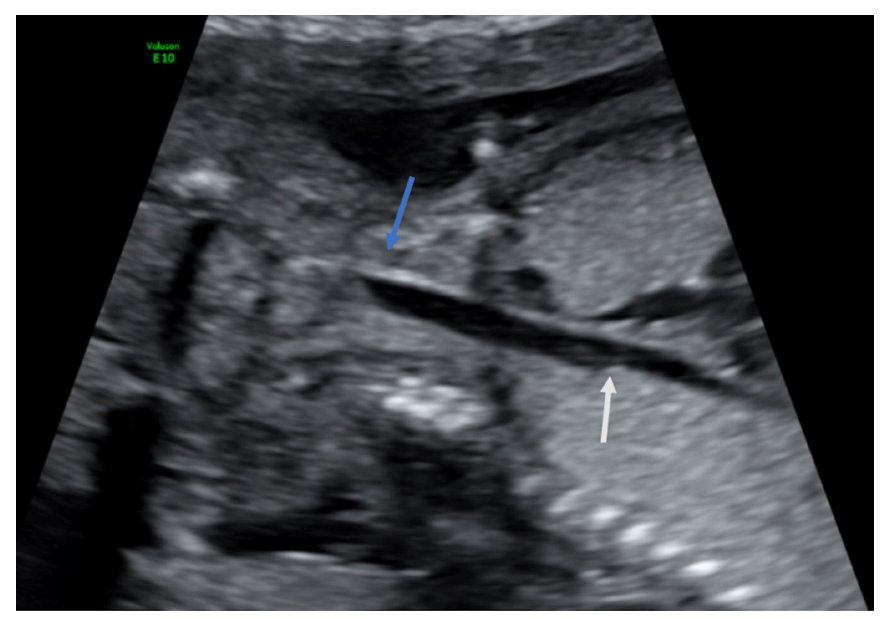

Figure 1 Ultrasound image with blue arrow pointing to the abrupt termination of the dilated trachea within the neck in the fetus with CHAOS. The white arrow is at the level of the trachea within the thorax surrounded by bilateral hyperechoic lung.

both nitroglycerin and terbutaline. Unfortunately, uterine relaxation was not sufficient to maintain optimal placental perfusion to the fetus. In an effort to expedite the procedure, the surgical team proceeded by placing a haemostatic myometrial box stitch at the fundus placed well away from the placental edge. The uterus was incised within the box stitch with electrocautery, and the amniotic sac was entered. Bainbridge clamps were used to compress the myometrium prior to placing uterine haemostatic staplers. Due to inadequate uterine relaxation, the thickened myometrium prohibited sufficient compression of tissue to allow for stapling. At this point, the uterine entry point was extended with electrocautery, and Allis clamps were used along the length of the hysterotomy to provide haemostasis. The fetal head and neck were then delivered followed immediately

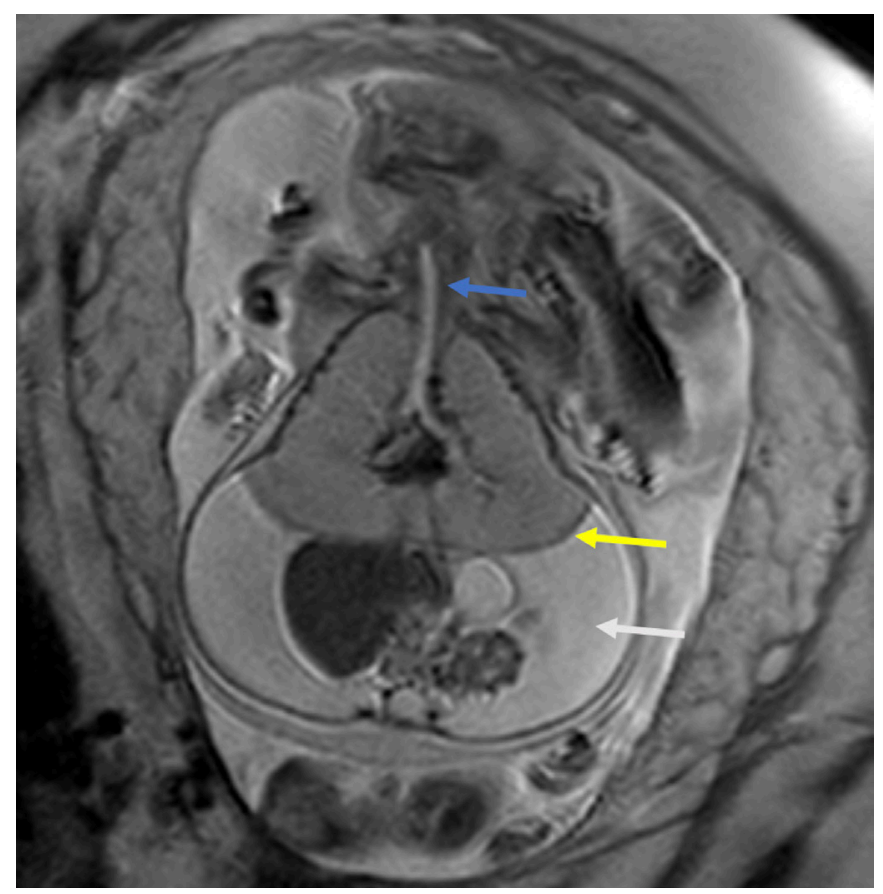

Figure 2 Fetal MRI showing the dilated fetal airway (blue arrow), everted diaphragm (yellow arrow) and ascites (white arrow) typical of fetal CHAOS.

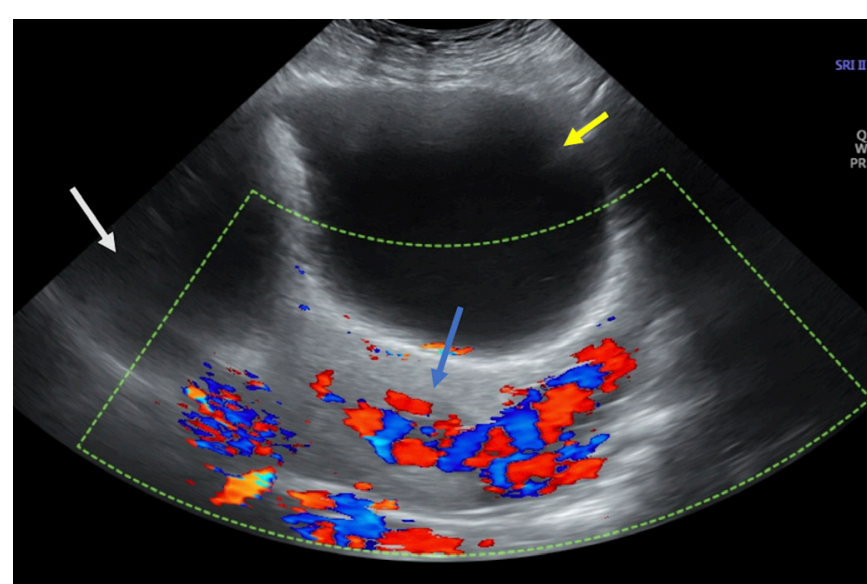

Figure 3 Colour Doppler on abdominal ultrasound demonstrating umbilical cord prolapse to within the vagina (blue arrow) at time of exit procedure, maternal lower uterine segment (white arrow) and maternal bladder (yellow arrow).

by an intramuscular injection of a cocktail containing atropine, rocuronium and fentanyl. A rapid intravenous infusion device was used to infuse warm lactated Ringer's solution through the hysterotomy to assist in maintaining uterine volume. Fetal monitoring performed throughout the procedure by fetal cardiology demonstrated intermittent bradycardia with rate as low as 88 beats/min and as high as 129 beats/min with depressed biventricular function. At this time, the paediatric surgeon and paediatric otolaryngologist worked in concert and established an airway via an endotracheal tube through a tracheostomy incision. The time from delivery of the head and neck until airway establishment was $3 \mathrm{~min}$. The remainder of the baby was delivered; the umbilical cord was clamped and cut; and the infant was passed off to the neonatology team.

The neonatal response to initial resuscitation was an immediate return to a heart rate in the 150's bpm with an oxygen saturation of $100 \%$. After stabilisation, bronchoscopy revealed laryngeal atresia below the level of the vocal cords with an intact trachea (figure 4).

The parturient was extubated after the placement of bilateral transversus abdominus plane blocks for postoperative pain control. Unfortunately, during the first 24 hours postpartum, the mother developed uterine atony necessitating the use of uterotonics and uterine balloon tamponade. She received a transfusion of 2 units of packed red blood cells. Subsequently, the mother remained inpatient for three postoperative days and was discharged in good condition without further complications from the surgical procedure.

\section{OUTCOME AND FOLLOW-UP}

The infant is doing well and remains with a tracheostomy awaiting definitive repair of the laryngeal atresia.

\section{DISCUSSION}

Despite meticulous planning, the EXIT procedure was performed in a suboptimal scenario secondary to multiple obstetrical factors. The patient presented to labour and delivery unscheduled on an early Sunday morning with a complex presentation including an umbilical cord prolapse with category II fetal heart rate tracing. In normal circumstances, an umbilical cord prolapse results in an emergent caesarean delivery. Because the fetus lacked a patent airway, an emergent caesarean would have resulted in a 

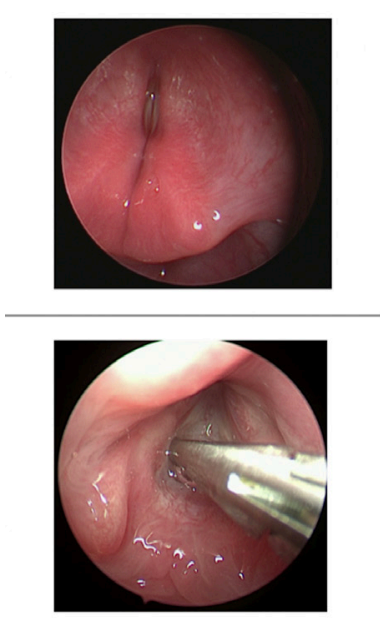

Figure 4 Upper left image demonstrates zoomed-in supraglottic view with normal anatomy with medialised vocal cords. Upper right image is the glottic view with medialised normal vocal cords. Bottom left image is the glottic view with cords spread demonstrating laryngeal atresia starting in the infraglottis. Bottom right image is the infraglottic view with laryngeal atresia.

neonatal demise or, in the best-case scenario, prolonged hypoxia during an attempt to establish an emergent airway immediately following delivery.

Maternal safety is of paramount importance when considering the option for an EXIT procedure. If maternal safety is compromised, the EXIT procedure should be abandoned to ensure an optimal maternal outcome. Fetal health follows second to the mother's well-being. Throughout this case, extensive monitoring of the patient's status was undertaken by both the anaesthesiology and surgical teams. Fetal compromise was certainly a concern with the non-patent airway, as well as an umbilical cord prolapse and suboptimal placental perfusion. Our team was able to assure maternal safety, and, therefore, the EXIT was allowed to proceed as planned but with notable deviations. Due to anhydramnios, cord prolapse, breech presentation and inadequate uterine relaxation, an external cephalic version and a low transverse uterine incision were not feasible, and access to the uterine fundus was needed to deliver the head and neck first. This necessitated a maternal midline incision sufficient to exteriorise the uterus to enable fundal access for the hysterotomy.

Once the uterus was exteriorised, uterine relaxation was attempted with an anaesthetic cocktail (halogenated agent, terbutaline and nitroglycerin) while maintaining stable maternal cardiovascular status with pressor infusions. Uterine relaxation was unfortunately never obtained despite maximal anaesthetic agents. As a result, the myometrial thickness compromised compression of the tissue with Bainbridge clamps, and use of the hysterotomy staples was rendered futile. Also, as a result of lack of uterine relaxation, optimisation of uteroplacental circulation was not achieved. Whether fetal bradycardia was secondary to the existing cord prolapse, increased uterine tone and/or loss of optimal uterine volume is not clear. Fortunately, there were no signs of placental abruption at any point, which would have terminated the EXIT procedure. Infusion of warm Ringer's lactate was not effective to maintain optimal uterine volume due to the myometrial contractile force in this circumstance, and the only volume maintained was secondary to the lower half of the fetus remaining within the uterus.
Fortunately, the tracheostomy was performed expeditiously without any issue. There was minimal time spent on uteroplacental bypass, which was present but suboptimal secondary to the aforementioned complicating factors. In addition, once the airway was secured, an awaiting neonatal team optimised oxygenation and ventilation.

Quality review performed after the procedure highlighted an important area for improvement, establishing the optimal gestational age to schedule an EXIT procedure. In one series of 31 EXIT procedures, the gestational age range at the time of delivery was quite variable at $29-40$ weeks. There is no existing consensus as to when timing is optimal. ${ }^{6}$ With the diagnosis of CHAOS, polyhydramnios is common and is associated with a high risk of premature rupture of membranes and premature labour. Our EXIT occurred at 34 weeks and 5 days. An amnioreduction could have been performed prophylactically to try to avoid this complication or the EXIT could have been scheduled earlier than the planned 37 weeks of gestation. Admission to the hospital is another consideration to maximise efficient movement to the operating room in the setting of spontaneous labour. In this case, the patient did have asymptomatic polyhydramnios with a long cervix, and the risks of a late amnioreduction was considered by the team to be higher than the benefits. Active labour following an amnioreduction can certainly occur. ${ }^{7}$ The benefit of waiting longer to promote fetal maturity was desired, although the ability to achieve this in the setting of CHAOS is unknown.

A critical component of the procedure is an established, experienced and highly collaborative multidisciplinary team within a quaternary care system. ${ }^{8}$ This asset cannot be understated and likely contributed to this case proceeding stepwise through the critical steps despite complicating factors and concluding with the efficient placement of a patent airway. The procedure was planned several weeks in advance and a walkthrough was performed with all key stake holders, which is standard protocol for every EXIT procedure at our institution. Simulation prior to each procedure results in a higher success rate, especially when it occurs unscheduled with few personnel on site. ${ }^{9}$

The literature on EXIT procedures primarily focuses on the technical aspects of the procedure and case series of its use for various fetal diagnoses, including CHAOS. Only in select few instances are maternal complications described and even fewer instances of cases where the EXIT procedure was abandoned prematurely due to maternal or fetal complications. The case series describe the fetal diagnosis and success or failure of establishing the airway. Maternal complications from EXIT procedures include longer operative times, higher blood loss and higher rates of wound complications compared with those undergoing caesarean delivery. ${ }^{210-12}$ In addition, uterine dehiscence and rupture in a subsequent pregnancy is a real risk. ${ }^{12}$ In an anaesthesia review of the largest case series of 65 patients, 32\% of cases occurred prior to the planned date for reasons including prelabour premature rupture of membranes, and $18 \%$ of cases were emergently performed. In $6 \%$ of the cases, the procedure was 'rushed' due to either identified placental abruption or what was identified as impending abruption. ${ }^{13}$ In these cases, as in ours, the definitive therapy was expeditious control of the fetal airway followed by delivery. Refractory elevated uterine tone is mentioned; however, the rate of this complicating factor is not known from the available literature, and there are likely many contributing factors. The key to success in these complicated cases appears to be the experience and the coordination of the surgical team. 
In summary, we have described an EXIT procedure complicated by significant obstetrical factors. In the absence of maternal complications, obstetrical complications, which can be multiple in nature, can be ameliorated and addressed such that an EXIT procedure can be successfully completed.

\section{Learning points}

- Lesser emphasis has been placed on obstetrical considerations during ex utero intrapartum therapy (EXIT) procedures; however, these factors may significantly affect the surgical procedure as well as the clinical outcome.

- In the absence of maternal complications and when maternal safety is ensured, an EXIT procedure may proceed despite significant obstetrical complications.

- An established and experienced multidisciplinary team with extensive surgical planning is essential for the successful completion of an EXIT procedure.

- A standard protocol for EXIT procedures should include a preoperative walk-through to rehearse all the critical steps for its successful completion.

Acknowledgements We thank Henry L Galan, Kenneth W Liechty and Nicholas Behrendt for their contribution in the surgical management of the patient, as well as in the preparation and review of this article.

Contributors MZ, MB and SCD, CF and CW contributed significantly to the conception and design, drafting and revision, as well as the final approval of this article, and are accountable to the accuracy and integrity of the article.

Funding The authors have not declared a specific grant for this research from any funding agency in the public, commercial or not-for-profit sectors.

Competing interests None declared.

Patient consent for publication Parental/guardian consent obtained.
Provenance and peer review Not commissioned; externally peer reviewed.

Open access This is an open access article distributed in accordance with the Creative Commons Attribution Non Commercial (CC BY-NC 4.0) license, which permits others to distribute, remix, adapt, build upon this work non-commercially, and license their derivative works on different terms, provided the original work is properly cited and the use is non-commercial. See: http://creativecommons.org/ licenses/by-nc/4.0/.

\section{REFERENCES}

1 Mychaliska GB, Bealer JF, Graf JL, et al. Operating on placental support: the ex utero intrapartum treatment procedure. J Pediatr Surg 1997;32:227-31.

2 Hirose $\mathrm{S}$, Farmer DL, Lee $\mathrm{H}$, et al. The ex utero intrapartum treatment procedure: looking back at the exit. J Pediatr Surg 2004;39:375-80.

3 Hoagland MA, Chatterjee D. Anesthesia for fetal surgery. Paediatr Anaesth 2017;27:346-57.

4 Marwan A, Crombleholme TM. The exit procedure: principles, pitfalls, and progress. Semin Pediatr Surg 2006;15:107-15.

5 Kohl T, Van de Vondel P, Stressig R, et al. Percutaneous fetoscopic laser decompression of congenital high airway obstruction syndrome (CHAOS) from laryngeal atresia via a single trocar--current technical constraints and potential solutions for future interventions. Fetal Diagn Ther 2009;25:67-71.

6 Bouchard S, Johnson MP, Flake AW, et al. The exit procedure: experience and outcome in 31 cases. J Pediatr Surg 2002;37:418-26.

7 Laje P, Peranteau WH, Hedrick HL, et al. Ex utero intrapartum treatment (exit) in the management of cervical lymphatic malformation. J Pediatr Surg 2015;50:311-4.

8 Osborn AJ, Baud D, Macarthur AJ, et al. Multidisciplinary perinatal management of the compromised airway on placental support: lessons learned. Prenat Diagn 2013;33:1080-7.

9 Pratt SD. Focused review: simulation in obstetric anesthesia. Anesth Analg 2012;114:186-90.

10 Moldenhauer JS. Ex utero intrapartum therapy. Semin Pediatr Surg 2013;22:44-9.

11 Noah MMS, Norton ME, Sandberg P, et al. Short-Term maternal outcomes that are associated with the exit procedure, as compared with cesarean delivery. Am J Obstet Gynecol 2002;186:773-7.

12 Zamora IJ, Ethun CG, Evans LM, et al. Maternal morbidity and reproductive outcomes related to fetal surgery. J Pediatr Surg 2013;48:951-5.

13 Lin EE, Moldenhauer JS, Tran KM, et al. Anesthetic management of 65 cases of ex utero intrapartum therapy: a 13-year single-center experience. Anesth Analg 2016;123:411-7

Copyright 2021 BMJ Publishing Group. All rights reserved. For permission to reuse any of this content visit

https://www.bmj.com/company/products-services/rights-and-licensing/permissions/

BMJ Case Report Fellows may re-use this article for personal use and teaching without any further permission.

Become a Fellow of BMJ Case Reports today and you can:

- Submit as many cases as you like

- Enjoy fast sympathetic peer review and rapid publication of accepted articles

- Access all the published articles

- Re-use any of the published material for personal use and teaching without further permission

Customer Service

If you have any further queries about your subscription, please contact our customer services team on +44 (0) 2071111105 or via email at support@bmj.com.

Visit casereports.bmj.com for more articles like this and to become a Fellow 